\title{
COMUNICACIÓN DIGITAL Y POLÍTICA: APROXIMACIONES PARA SU GESTIÓN
}

\author{
DIGITAL COMMUNICATION AND POLICY: approaches to its management
}

POLÍTICA DE COMUNICAÇÃO DIGITAL E: abordagens de gestão

\author{
Apolo Diego ${ }^{1}$ \\ Guerrero Sofía ${ }^{2}$ \\ Jiménez Xavier ${ }^{3}$ \\ Universidad de Las Américas, Ecuador \\ diego.apolo@udla.edu.ec \\ sofía.guerrero@udla.edu.ec \\ xavier.jimenez@udla.edu.ec
}

\begin{abstract}
${ }^{1}$ Docente Carrera de Comunicación Corporativa, Facultad de Comunicación y Artes Audiovisuales, Universidad de las Américas, Quito-Ecuador. Ponente invitado en congresos de reconocido prestigio internacional, ha publicado libros, capítulos y artículos en revistas indizadas, además de realizar investigaciones, se ha desempeña como docente en la Universidad de las Américas Quito-Ecuador. Es considerado candidato a Doctor en la Universidad Nacional de La Plata - Argentina, posee una Maestría en Comunicación Estratégica en la Pontificia Universidad Católica de Chile y ha trabajado durante los últimos 7 años en consultorías nacionales e internacionales en organizaciones privadas, públicas y del tercer sector.

2 Docente Carrera de Comunicación Corporativa, Facultad de Comunicación y Artes Audiovisuales, Universidad de las Américas, Quito-Ecuador Master en Ciencias Políticas de la Universidad de Idaho en Estados Unidos y en Proyectos de Paz y Mediación - Universidad de Chulalongkorn, Tailandia. Tiene un diplomado Responsabilidad Social Empresarial - Tecnológico de Monterrey y es licenciada en Comunicación con mención en Periodismo de la PUCE. Su experiencia profesional incluye trabajos en el campo de comunicación, responsabilidad social, políticas públicas y sostenibilidad. Actualmente, se desempeña como docente de la Carrera de Comunicación Corporativa de la UDLA y consultora en América Latina. Adicionalmente, ha recibido entrenamiento especializado en Estados Unidos, Brasil, México y Asia del Este.

${ }^{3}$ Docente Carrera de Comunicación Corporativa, Facultad de Comunicación y Artes Audiovisuales, Universidad de las Américas, Quito-Ecuador. Comunicador a tiempo completo. Master Internacional en dirección de Comunicación Empresarial a Institucional. Productor Audiovisual y Multimedia. Cuenta con 6 años de experiencia profesional. Es Docente de Apolo Académico en la Carrera de Comunicación Corporativa de la Universidad de las Américas y Consultor en comunicación de Nilam Negocios sostenibles y QuimaTv. Se ha desempeñado profesionalmente en proyectos y campañas de comunicación para farmacéuticas e instituciones públicas de salud y de la banca del Ecuador.
\end{abstract}

REDMARKA UIMA-Universidad de A Coruña - CIECID

Año VIII, Número 15, (2015), v I pp. 3-22

http://www.redmarka.net/ ISSN 1852-2300 
Material original autorizado para su primera publicación en la revista académica REDMARKA. Revista Digital de Marketing Aplicado.

https://doi.org/10.17979/redma.2015.01.015.4874

Recibido: 17 Noviembre 2015

Aceptado 4 Diciembre 2015

\section{Resumen:}

Con el desarrollo de las Tecnologías de Información y Comunicación se evidenció un desarrollo tecnológico que ha modificado comportamientos culturales en las relaciones político sociales; las mismas que han dado un nuevo frente de comunicación directa para la interacción entre los usuarios de internet y gobernantes. Esto ha requerido de espacios donde los ciudadanos demanden acciones, procesos de rendición de cuenta y transparencia en la gestión pública.

Frente a ésta necesidad aparece como espacio de articulación el e-gobierno, como una forma de optimizar el proceso analítico sistemático para la gestión de comunicación digital en política, resaltando sus acciones y evitando su instrumentalización, la misma que brinda espacios digitales de participación ciudadana que se complementan con el uso de la web 2.0, como un canal multidireccional que genera y fomenta vínculos y relaciones.

\section{Abstract:}

The development of Information and Communication Technologies has demonstrated a technological expansion that changed the cultural behavior in social political relations giving a new space for interaction between Internet prosumers and rulers. This phenomenon required spaces where citizens are able to demand actions, processes of accountability and transparency in governance.

Additionally, the e-government performs as a new way to enhance the systematic analytical processes to manage digital communication policies highlighting their 
actions and preventing their mistreatment. This springs a digital opportunity for citizen participation which can be complemented through the use of Web 2.0, as a multi-channel to generate and foster links and relationships.

\section{Resumo:}

O desenvolvimento das Tecnologias de Informação e Comunicação tem demonstrado uma expansão tecnológica que mudou o comportamento cultural nas relações políticas sociais dando um novo espaço de interação entre prosumers, Internet e governantes. Este fenômeno espaços necessários onde os cidadãos são capazes de exigir ações, processos de prestação de contas e transparência na governação.

Além disso, o e- governo executa como uma nova maneira de melhorar os processos para gerenciar políticas de comunicação digital destacando suas ações e prevenir maus tratos. Esta brota uma oportunidade digital para a participação do cidadão, que pode ser complementada através do uso de Web 2.0, como um multicanal para gerar e laços e relações adotivos.

\section{Palabras Claves:}

Comunicación digital, política, e-gobierno, participación ciudadana, internet

\section{Keywords:}

Digital communication, politics, e-government, citizen participation, internet.

\section{Palavras chave:}

Comunicação digital, política, governo eletrônico, a participação do cidadão, internet. 


\section{Introducción}

Al ser la comunicación un proceso fundamental en la interacción social, Wilcox y otros autores (2001) mencionan que sus funciones son: informar, persuadir, motivar y lograr la comprensión mutua. Por esta razón, es relevante conocer la manera en que se constituyen los procesos de comunicación, la forma en que los mensajes son percibidos, cómo se procesa dicha información y qué tipos de medios y herramientas de comunicación son los más adecuados para generar vínculos con stakeholders.

Al hablar de política, es inminente involucrar y destacar el rol que cumple la comunicación, al ser un eje transversal que permite acercarse a los grupos de interés logrando fomentar sistemas de participación ciudadana que se convierten en la base de la democracia. "La política es comunicación" (Canel, 2006:17), desde los aportes de Noguera "la política es un acto comunicativo referido al poder público (...) y lo es, en cualquiera de sus instancias: creación, regulación y desarrollo" (2008:127) de contenidos que generarán opinión pública. En este sentido, la política se relaciona con los diferentes medios de comunicación y la influencia que ésta ejerce sobre los ciudadanos.

Sin embargo, esta percepción no puede ubicarse en la plataforma social como una lucha por parte de políticos por ocupar un espacio público, sino como una forma de análisis estratégico que permite recurrir a los medios de comunicación como herramientas que generan un acto comunicativo que permite dialogar con las preferencias ciudadanas, lo cual lleva a crear estrategias; ya que, desde el espacio público se construye la opinión pública a través de cada conversación que realizan los electores desde su cotidiano y vida social (Habermas, 1973 en Boladeras, 2001).

Las instituciones políticas han recurrido a estos espacios para generar vínculos e información que les permita alcanzar sus objetivos, tomando en cuenta que con avance tecnológico "ahora el ciudadano dispone de información internacional en su 
casa con un pequeño ordenador personal, se comunica en tiempo real con cualquier país del mundo" (Yanez, 2007:365).

\section{Un recorrido por el desarrollo web}

Las constantes innovaciones de las Tecnologías de Información y Comunicación (TIC), han marcado pautas en cuanto a la "economía, la sociedad o la cultura" (Anduiza y otros, 2010:7); en este sentido, es relevante en primera instancia brindar aproximaciones que permitan identificar dos etapas por las cuales ha atravesado la Web, tomando en cuenta que antes el aparecimiento de las TIC "las posibilidades de compartir información entre distancias lejanas resultaban escasas, se requerían grandes esfuerzos para conectar a las sociedades" (Aliaga, 2005:4). La primera etapa fue el desarrollo web (1.0) que tuvo como base la estructura codificada en donde los primeros encargados de gestionarla fueron ingenieros en sistemas con conocimiento en programación, esto provocaba atadura, énfasis en los códigos y comunicación unidireccional por parte de instituciones.

Con la prematura inserción del lenguaje codificado, Hyper Text Markup Lenguaje (HTML), usado en primera instancia por el Departamento de Defensa de los Estados Unidos como lo expone Hobbes (1997), sus desarrolladores no se imaginarían el gran despliegue tecnológico al que dieron inicio y los efectos socio culturales que causarían a nivel mundial.

Con el nacimiento de la primera gran red, Advanced Research Projects Agency Network (ARPANET), y la concatenación del HTML, las instituciones militares lograron generar y mantener el primer tipo de comunicación digital en red donde "los modos de tratar los signos no verbales, independientemente del resultado de la interacción a la cual se "presenta" la persona en cada situación" (Bernández, 2013:11), son cada vez más interactivos y mediados por diferentes dispositivos. 
En una segunda etapa, apareció el desarrollo web (2.0), donde el principal enfoque fue la interacción y autogestión; de esta manera, un gran aporte fueron los sistemas de gestión de contenidos o Content Management System (CMS) como: Joomla, Wordpress, Drupal, entre otros, los mismos que rompieron la atadura de la programación para el desarrollo web, abriendo su manejo para que diferentes profesionales puedan emplear estas nuevas plataformas para desarrollar estrategias que permitan el acercamiento de las instituciones hacia los usuarios.

Bajo la lógica del desarrollo web y sin caer en determinismos tecnológicos, es necesario recalcar que la comunicación y la política también fueron impactadas por el uso masificado de las Tecnologías de Información y Comunicación; la amplitud e inmediatez con que surgieron éstos cambios dejaron en evidencia por ejemplo que mientras a la radio le llevó 38 años alcanzar una audiencia de 50 millones de oyentes; y a la televisión 14 años para lograr el mismo número de televidentes, internet necesitó solamente cuatro años para ello y hoy las plataformas de redes sociales como "Facebook o Twitter, superaron ampliamente esas audiencias al poco tiempo de su lanzamiento" (Lanza, 2011:54) y sobre todo desde los aportes de Aladro, Valbuena y Padilla tener "claro que todos estos nuevos sistemas de comunicación harán revolucionar las posibilidades de desarrollo, crecimiento e integración social" (2012:38) de niños, jóvenes y adultos.

La innovación tecnológica instrumental-artefactual permitió el advenimiento de comunidades, portales y centros de recursos bajo la misma lógica de los CMS, en donde la promulgación de redes sociales y otros recursos fueron creciendo en base a la colaboración global, aprovechando "el contenido simbólico de los mensajes" (Thompson, 1998:27) que circula en dichas plataformas permitiendo observar nuevos espacios de convivencia y la representación de una vida digital, en donde Bustamante (2014:23), recurre a la Organización de Estados Americanos para mencionar que ésta "habilidad para mezclar cultura y tecnología, en la creación, en los productos y en los mercados parece emerger como una dimensión clave de la 
vida económica globalizada", por lo cual la comunicación debe dialogar con otras áreas afines como la publicidad, el marketing entre otras, permitiendo reforzar las estrategias más allá de la lucha por las preferencias ciudadanas en campañas electorales sino permitir el diálogo "entre gobierno y gobernados a lo lardo de periodos no electorales" (Juárez, 2003:68).

\section{Espacios de interacción: comunicación digital y política}

Con estas aproximaciones brindadas sobre el desarrollo web y el innegable vínculo gobierno y ciudadanía mediado por comunicación digital, es importante insistir que en la actualidad nos relacionamos en una sociedad hiperconectada, la cual está compuesta por prosumidores, con nuevas exigencias en donde instituciones deben "adaptarse a la nueva ecología impuesta por los medios digitales" (Renó y otros, 2014: 71) además que se debe "comprender que para llevar a un llamado a la acción del consumidor digital, el valor fundamental de todo el trabajo está en la investigación y el relacionamiento" (Apolo y otros, 2015:17).

Este desarrollo a gran escala permitió la aparición de nuevos canales/medios como el correo electrónico, blogs, chats, mensajería instantánea, redes sociales, entre otras aplicaciones que acapararon la atención de la ciudadanía como un nuevo espacio de participación. Este contexto implica procesos de investigación, uso, adaptación y comunicación mediante la web 2.0, las instituciones y/o figuras públicas al emplear en sus estrategias la comunicación en espacios digitales, les permite conformándose como líderes de opinión aprovechando éstos espacios para el ocio, información, comunicación, debate, generación y articulación de contactos y en muchas ocasiones la generación de opinión pública. 
La gestión de comunicación con un enfoque estratégico permite marcar una ruta hacia la competitividad y la eficiencia, estableciendo medidas que permitan la fácil adaptación al cambio (Gálvez, 2014), es así como desde estos aportes, el manejo de web 2.0 y política ofrece grandes oportunidades de diálogo, debate y espacios para la promulgación de democracia, permitiendo "la transmisión de la opinión de los ciudadanos de una manera más simple, más rápida y más directa, fomentando la creación de unas instituciones públicas mejor informadas, más innovadoras, más centradas en el ciudadano y, por tanto, más democráticas" (Criado y Rojas. 2015:35). En síntesis, la comunicación política en entornos 2.0 permite la participación descentralizada, deslocalizada, inmediata por su interfaz de usuario gráfica y estructura consiguiendo:

“(i) generar espacios de debate y concienciación ciudadana, más plurales y representativos, disminuyendo la asimetría de la información, (ii) contribuye a la prestación de servicios públicos con mayor eficiencia y eficacia, y (iii) constituye un nuevo medio accesible, de fácil uso y con mucha penetración, que ayuda al gobierno a la difusión de la información sobre su gestión, las políticas públicas, las actividades desarrolladas y los proyectos por ejecutar en los distintos ámbitos de interés para la comunidad, favoreciendo los procesos de transparencia y rendición de cuentas" (Criado y Rojas. 2013: 45).

Estos factores permitirán la formación de una imagen política en la percepción de los usuarios, ahora bien, la web 2.0 es considerada como un recurso público" (Orihuela. 2014:44) la misma que responde a la siguiente estructura para su concepción:

1. Generar un perfil,

2. Articular usuarios o nodos,

3. Establecer relaciones con los actores. 
La utilización de la web 2.0 no es reciente en la gestión de comunicación en política, se debe tomar en cuenta que desde el arranque y masificación 2005-2007, los políticos han implementado dentro de sus estrategias en campañas electorales su uso y aplicación para generar espacios de debate. Un ejemplo claro es la eclosión de las redes que representó un fenómeno para la comunicación política en Estados Unidos como la estrategia de relacionamiento aplicada por el actual Presidente Barack Obama quien logró recaudar una gran cantidad de fondos monetarios a más de darse a conocer y posicionar su imagen en el cotidiano de los electores.

En este sentido y considerando las ventajas de la comunicación digital, es importante considerar la siguiente interrogante ¿el administrador de una institución está preparado para la gestión de comunicación en política internet?; es así cómo "cada institución es la encargada de responderse qué desea generar con su presencia digital" (Apolo, 2014:37), considerando que para la incorporación de las redes sociales dentro de los mecanismos de comunicación a disposición de los públicos es vital identificar la capacidad y nivel de respuesta, esta primera instancia obligaría a pensar si realmente está o no preparado para asumir la incorporación de canales 2.0, como conocemos gracias los aportes y literatura, la gestión en entornos digitales necesita un proceso estratégico, ético e integral que toda administración debería disponer "de un canal de comunicación multidireccional con la ciudadanía -con empresas y entidades inclusive- sin intermediarios que le permite recibir el feedback instantáneo de sus actuaciones y propuestas." (Criado y Rojas. 2013: 10).

En este sentido es importante considerar que la gestión de redes sociales dentro de la administración y política respondería a la capacidad de respuesta y planificación "cuando las acciones en comunicación tienen carácter permanente, es necesario que atraviesen un proceso de planificación, es decir, una investigación previa, cuyos resultados han de ser estudiados y formulados en términos de lineamientos a futuro" (Prieto, 1999:106), tomado en cuenta que "la tecnología de la web va a 
impulsar nuestro futuro y a tratar de replicarnos por completo - dice Krotosky-. Pero no como se ve en Blade Runner (Ridley Scott, 1982), sino que va a proporcionar un servicio a las necesidades humanas basada en nuestros intereses y en cómo interactuamos en el ciberespacio" (Albarello, 2012:3).

\section{E-gobierno y participación ciudadana.}

A partir de dichos avances los gobiernos han visto en estos nuevos medios, la oportunidad para generar una democracia digital participativa con la ciudadanía que dé respuesta a las necesidades expresadas en diferentes espacios que hoy se han convertido en lugares de encuentro para la participación política. Este lineamiento provocó reflexiones con respecto a e-gobierno o gobierno electrónico como una forma para demostrar una administración más receptiva y transparente. Izurieta, Perina y Arterton (2003:176) afirman que el desarrollo de e-gobierno "provocará que los medios de comunicación tradicionales cuenten con información detallada sobre cualquier política de gobierno y permitirá al político y al público tener participación activa ante los medios".

Con los avances de la web 2.0 el concepto de e-gobierno se ido constituyendo "con el fin de facilitar la aplicación de prácticas de buen gobierno que contribuyan a incrementar la transparencia informativa de la sociedad" (Gandía y Pérez, 2005:14). Con este sistema electrónico se podría también agilitar trámites y servicios que ofrece el Estado a la población; y de esta forma se promovería la desburocratización al brindar servicios más ágiles y mejores que tengan un menor costo para las instituciones de gobierno. Sin embargo, los servicios digitales deben pasar por un proceso de familiarización que permita romper las barreras digitales entre sus usuarios. Retomando aportes de Izurieta, Perina y Arterton (2003:177178), algunas de las características que el e-gobierno ofrece son:

"Agrupar y exponer trabajos, publicaciones, informes, presentaciones y datos de interés acerca del gobierno, de sus instituciones y funciones. 
Poner a disposición del público una base actualizada de leyes.

Promocionar la información adecuada sobre la gestión del presidente de la República y sus actividades en el ámbito de gobierno.

Presentar una guía referencial e informativa del sector público, que contemple los servicios públicos que ofrece, los cuales pueden ser gestionados vía electrónica.

Promocionar un servicio de correo informativo, que distribuya periódicamente las leyes, datos, noticias y varios temas del Estado.

Facilitar a los ciudadanos algunos servicios tales como: declaraciones de impuestos, bolsa de empleo, entre otros"

Según Ramos "los gobiernos tienen la necesidad de comunicarse principalmente con los ciudadanos, para conservar su legitimidad en el ejercicio del poder" (Ramos, 2008:34). Con el e-gobierno se procura que los servicios electrónicos sean más abiertos a las necesidades de los ciudadanos y las empresas; permitiendo el acceso a la información pública y facilitando una serie de gestiones, trámites y servicios a través del internet tomando en cuenta la necesidad de la coordinación entre entidades gubernamentales y sus codependencias hacia la gestión eficiente, tomando en cuenta como lo menciona Ulloa (2007:49) que "para que haya comunicación no solo se necesita de dos o más interlocutores es el proceso, sino de un espacio adecuado que los preste las condiciones más válidas y cómodas de interacción para que el intercambio de ideas fluya con soltura"; es decir no centrarse únicamente en el desarrollo del sistema sino en la usabilidad que podrían darle los usuarios.

Es así cómo el e-gobierno permite gestionar mejoras en la gobernabilidad y en el servicio público; desarrollando derechos de participación y logrando interacción entre los gobiernos y los ciudadanos; utilizando "a los medios masivos y alternativos para diseñar campañas no convencionales que generen una verdadera 
comunicación y una verdadera interacción con cada uno de los votantes" (Dávalos, 2011:2).

La comunicación interactiva permite adicionalmente fomentar la inteligencia colectiva y el aprendizaje colaborativo generando estrategias y acciones para que gobierno y servicios públicos dialoguen con los ciudadanos, siendo relevante "tener en cuenta al público destinatario al que queremos llegar" (Giansante, 2015:55) vinculando los contextos sociales al sistema político a través de un método que acompañe los objetivos plateados (Giones, 2010).

Es de ésta manera como procesos de participación ciudadana pueden ser analizados desde una mirada comunicacional vinculada a los avances tecnológicos; ya que, éstos espacios públicos se conforman desde las nuevas comunidades digitales que llevan consigo las antiguas connotaciones de las "plazas, bandejones, parques, calles, veredas y avenidas" (Domínguez, 2000:81), en donde los ciudadanos buscan su apropiación que hoy es mediada por pantallas y una presencia digital, modificando el cotidiano de los sujetos y sus relaciones; así, dichos avances tecnológicos permiten observar a la participación como "un componente esencial de la democracia, como forma de organización social y de gobierno" (Ziccardi: 2000:2) que debe tomar en cuenta los "contextos sociales particulares y a partir de capitales culturales y simbólicos acumulados en determinadas condiciones sociales" (Benítez, 2013:227) que permitan articular procesos de intervención que respeten y fomenten la apropiación de éstos.

Estos nuevos espacios de participación son un terreno en co construcción donde se hace necesario que los encargados de la gestión de comunicación de instituciones públicas deban estar presentes "en ellas para acercarse donde se encuentra la ciudadanía" (Criado y Rojas, 2013:22), reconociendo sus espacios e interacciones con el fin de que estos sean considerados como redes de integración y participación política propositiva. 
Estos procesos deben ir acompañados de la gestión de identidad digital entendida "como la habilidad de gestionar con éxito la propia visibilidad, reputación y privacidad en la red como un componente inseparable y fundamental del conjunto de habilidades informacionales y digitales" (Giones, 2010:2) y darles su importancia al momento de gestionar y fortalecer relaciones sociales, además de aprovechar la penetración y apropiación que brindan estos espacios, donde los aportes brindados por McLuhan (1972) y Toffler (1990) evidencian que la práctica digital lleva consigo vinculada la capacidad de ser productores y consumidores (prosumidores) de contenidos reconociendo esa "posibilidad política, comunicativa, cultural y tecnológica" (Rincón, 2008:93), que tienen los usuarios como potenciales generadores de comunicación interactiva local-global (Jenkins, 2008) abriendo la posibilidad para pensar a dichas relaciones como espacios reticulares que estén en constante construcción y aporte desde la tecnología; ya que, ésta "transforma los modos de relacionarnos, informarnos, educarnos, entretenernos y hacer negocios" (Orihuela y Santos, 2004:397).

Es así como un pilar fundamental para el diálogo entre los avances tecnológicos y participación ciudadana es comprender que las nuevas formas de construir una identidad corporativa desde una filosofía y cultura (Capriotti, 1999) estableciendo procesos participativos, y en el caso de instituciones públicas cuestionarse la manera de integrar estos enfoques para su diseño y gestión de comunicación en política.

\section{Redes sociales: un espacio para la relación entre ciudadanía y gobiernos}

Navarro y Salazar brindan aportes relevantes para estructurar un análisis de redes sociales en donde las "relaciones y flujos entre actores tales como personas, grupos, organizaciones $u$ otras entidades procesadoras de información y/o conocimiento" (2007:70), sean tomadas en cuenta para detectar nodos en base a 
vínculos que permitan generar relaciones y en éste caso fortalecer mediante el manejo de redes sociales la interacción entre gobiernos y ciudadanos.

Para identificar los procesos en avances tecnológicos y participación, es relevante partir de la premisa propuesta por Bronstein y su equipo quienes comprenden que "toda organización social es una red cognitiva" (1994:380), que va adquiriendo diferentes formas y estructuras funcionales en base a sus procesos cotidianos, dichos procesos están entrelazados mediante nodos, vínculos y conversaciones que las cruzan y las constituyen, continuando con ello mencionan que "definir a una organización social como red cognitiva implica:

1. Hacer referencia a cierta tipología particular que caracteriza a una organización social, donde los nodos están definidos por individuos o por conjuntos de individuos que conforman a su vez una red que opera como subsistema de la red global; y donde las conversaciones constituyen los elementos que relacionan estos nodos.

2. Remitir a ciertas analogías que se puede establecer con otras redes cognitivas naturales (el sistema nervioso y el sistema inmunológico principalmente)" (Bronstein y otros, 1994:382).

Así, el poner en práctica estrategias que fortalezcan los vínculos entre el gobierno y los ciudadanos, en base a la participación obligan a que representantes de instituciones políticas y encargados de la gestión de comunicación en política deban reflexionar y "aprender nuevas habilidades y competencias cognitivas, digitales y de uso de los medios de comunicación y los recursos tecnológicos". (Ibarra y Llata, 2010:6), teniendo en cuenta para su aplicación que éstas:

- Fortalecen y favorecen el intercambio de comunicación.

- Suprimen la necesidad de coincidir en tiempo y espacio.

- Eliminan la linealidad en la comunicación.

- Exponen información con la aplicación de diferentes recursos.

- Permiten desarrollar trabajos colaborativos. 
- Implican nuevos espacios para generar opiniones y discusión.

\section{Conclusiones:}

La política no puede dejar a un lado la comunicación porque es un eje transversal que articula procesos que vinculan a los gobiernos y ciudadanos, evidenciando en sí espacios democráticos que deben buscar la eficiencia en gestión gubernamental y la participación en espacios públicos con el aprovechamiento de nuevos canales.

El e-gobierno se ha convertido en la nueva forma del espacio público que permite a las dependencias políticas interconectarse y establecer vínculos "en dirección al usuario" (Cusot y Klein, 2015:12); con el fin de agilitar los servicios y comunicar sus avances a través del fortalecimiento de los procesos democráticos, posicionando de ésta manera su gestión gubernamental y la de sus representantes.

Las reflexiones presentadas permiten reconocer la manera en que la gestión de comunicación digital posee un enfoque analítico estratégico y es un eje fundamental para los procesos políticos, en donde los gobiernos deben buscar vínculos con los ciudadanos que permitan el reconocimiento de procesos sociales del entorno, además de observar que la gestión de e-gobierno, tiene que ser un referente en la propuesta de planes de rendición de cuentas fomentando participación ciudadana y transparencia.

Finalmente, se debe subrayar que en la actualidad el poder político ha aprovechado la web 2.0 para acercarse a los ciudadanos, proveyendo servicios que agiliten su labor y permitan un libre acceso a la información y gestión de contenidos, es relevante por ende desde las perspectivas presentadas; reconocer a la comunicación como un proceso analítico sistemático que no se lo puede reducir únicamente a una instrumentalización y a la generación de contenidos unidireccionales. 


\section{Bibliografía:}

Aladro, E., Valbuena, F., y Padilla, G. (2012). Redes sociales y jóvenes universitarios españoles: nuevos ejes de socialización. Austral Comunicación. 1 (1), 27-40.

Albarello, F. (2013). La revolución virtual: riesgos y desafíos. Austral Comunicación. $1(2), 1-6$.

Aliaga, F. (2005). La construcción democrática del conocimiento tecnológico: una visión sociológica del software libre. Aposta: Revista de ciencias sociales, (20), 1-17.

Anduiza, E.; Cantijoch, M., Gallego, A., y Salcedo, J. (2010). Internet y participación política en España. Madrid: Opciones y actitudes (63). Centro de investigaciones sociológicas.

Apolo, D. (2014). No todo número es un dato: el mea culpa de las instituciones en redes sociales. Revista Dircom. (104), 37.

Apolo, D., Altamirano, V., Vásconez, V. y Cevallos, M. (2015). Usuarios, clientes y consumidores digitales: consideraciones para su abordaje desde el marketing y la comunicación corporativa. Redmarka: revista académica de marketing aplicado, (14), 3-19.

Benítez, S. (2013). Lo popular a partir de la apropiación de las TIC. Tensiones entre representaciones hegemónicas y prácticas. Question, 1 (38), 215-229.

(Recuperado el día 25 del mes 07 de 2015, de http://perio.unlp.edu.ar/ojs/index.php/question/article/view/1813/1575).

Bernández, M. (2013). Enfoques desde la producción audiovisual. Buenos Aires: La Crujía Ediciones.

Boladeras, M. (2001). La opinión pública en Habermas. Anàlisi: quaderns de comunicació i cultura, 26 (51-70). (Recuperado el día 28 del mes 06 de 
2015, de

http://ddd.uab.cat/pub/analisi/02112175n26/02112175n26p51.pdf).

Bronstein, V.; Gaillard, J. y Piscitelli, A. (1994). La Organización Egoísta. Clausura Operacional y Redes Conversacionales. En Delgado, J.; y Gutiérrez, J. (eds.). Métodos y Técnicas Cualitativas de Investigación en Ciencias Sociales. Madrid: Editorial Síntesis S.A.

Bustamante, E. (2014). Comunicación y cultura en la era digital, Industrias, mercados y diversidad en España. Barcelona: Editorial Gedisa.

Canel, M. (2006). Comunicación Política, una guía para su estudio y práctica. Madrid: Tecnos.

Castillo, D. (1999). Tres experiencias de diagnóstico de comunicación. Quito: CIESPAL.

Criado, J. y Rojas, F. (2013). Aproximación general sobre la adopción y uso de las redes sociales en las administraciones públicas. En. Criado, J. y Rojas, F. (eds.). Las redes sociales digitales en la gestión y las políticas públicas. (16-31). Barcelona: Escola d'Administració Pública de Catalunya.

Cusot, G. y Klein, C. (2015). Redes colaborativas: Wikipedia ¿Es confiable? Estrategas: investigación en Comunicación. (2), 81-92.

Dávalos. A. (2011). ¿Cómo diseñar campañas políticas exitosas? II Cumbre mundial de Comunicación Política. Quito.

Domínguez, V. (2000). Aspectos normativos e institucionales. En Segovia, O. y Dascal, G. (2000). Espacio público, participación y ciudadanía. (80-85). Santiago de Chile: Ediciones Sur.

Gálvez, N. (201). Comunicación estratégica: el plus en la gestión empresarial. Estrategas: investigación en Comunicación. (1), 19-28.

Giansante, G. (2015). La Comunicación política online. Madrid: Editorial UOC. 
Giones-Valls, A., \& i Brustenga, M. S. (2010). La gestión de la identidad digital: una nueva habilidad informacional y digital. BiD: textos universitaris de biblioteconomia i documentació, juny, núm. 24. (Recuperado el día 08 del mes 07 de 2015, de http://bid.ub.edu/24/giones2.htm).

Habermas, J. (1973). Öffentlichkeit (ein Lexikonartikel) 1964 y Die Geschichte von denzwei Revolutionen ( $H$. Arendt). En Kultur und Kritik. Francfort:

Suhrkamp. (Recuperado el día 05 del mes 08 de 2015, de http://www.bildung.unisiegen.de/berufspaedagogik/lehre/downloads/habermas_rolle_2.pdf).

Hobbes, R. (1997). Hobbes' Internet Timeline 12. Zakon Group LLC and OpenConf. (Recuperado el día 19 del mes 08 de 2015, de http://www.zakon.org/robert/internet/timeline).

Ibarra, A. y Llata, D. (2010). Niños nativos digitales en la sociedad del conocimiento: acercamientos conceptuales a sus competencias. Razón y Palabra. (72), $1-24$.

Izurieta, R., Perina, R., \& Arterton, C. (Eds.). (2003). Estrategias de comunicación para gobiernos. Buenos Aires: La Crujía.

Jenkins, H. (2008). Convergence Culture. La cultura de la convergencia de los medios de comunicación. Barcelona: Paidós.

Juárez, J. (2003). Hacia un estudio del marketing político: limitaciones teóricas y metodológicas. Espiral. Estudios sobre Estado y Sociedad. 9 (27), 61-95.

Lanza, L. y Fide, N. (2011) Política 2.0 y la comunicación en tiempos modernos. Cuadernos del Centro de Estudios en Diseño y Comunicación. Ensayos. (35), 53-63. (Recuperado el día 04 del mes 08 de 2015, de http://www.scielo.org.ar/scielo.php?script=sci_arttext\&pid=S185335232011000100006). 
McLuhan, M., y Nevitt, B. (1972). Take today: The executive as dropout. New York: Harcourt Brace Jovanovich.

Navarro, L., y Salazar, J. (2007). Análisis de redes sociales aplicado a redes de investigación en ciencia y tecnología. Sínt. Tecnol. 3(2), 69-86.

Orihuela, J. y Santos, M. (2004). Comunicar para crear valor. La dirección de comunicación en las organizaciones. Barcelona: EUNSA.

Orihuela, J. (2014). 80c claves sobre el futuro del periodismo. Barcelona: Ediciones Anaya Multimedia.

Prieto, D. (1999). Tres experiencias de diagnóstico de comunicación. Quito: CIESPAL.

Ramos, C. (2008). Comunicación comunitaria: cuadernos de capacitación electorales N-5. Quito: Instituto de la democracia.

Renó, D.; Camplans, C.; Ruiz, S. y Gosciola, V. (2014). Periodismo Transmedia: Miradas Múltiples. Bogotá: Editorial Universidad del Rosario.

Rincón, O. (2008). No más audiencias, todos devenimos productores. Comunicar: Revista científica iberoamericana de comunicación y educación. (30), 9398.

Thompson, J. (1998) Los media y la modernidad. Barcelona: Paidós.

Toffler, A., \& Martín, A. (1990). La tercera ola. Bogotá: Plaza \& Janes.

Ulloa, C. (2007). Comunicación, cultura y desarrollo. Quito: Quipus.

Wilcox, D.; Autt, P.; Agee, W. y Cameron, G. (2001): Relaciones Públicas.

Estrategias y Tácticas, Madrid: Pearson Educación.

Yanez, R. (2007). La comunicación política y los nuevos medios de comunicación personalizada. Ámbitos: revista internacional de comunicación. (16), 355365. 
Ziccardi, A. (2000), Los actores de la participación ciudadana. Instituto de Investigaciones Sociales, Universidad Nacional Autónoma de México. (Recuperado el día 30 del mes 07 de 2015, de http://controlatugobierno.com/archivos/bibliografia/ziccardiactoresparticipa cion.pdf). 